\author{
Karim Asehnoune \\ J. Claude Hemphill III \\ Rachel S. Agbeko
}

\section{Visualizing secondary brain insults: does the emperor have new clothes?}

Received: 24 April 2015

Accepted: 30 April 2015

Published online: 3 June 2015

(C) Springer-Verlag Berlin Heidelberg and ESICM 2015

\section{K. Asehnoune ( $)$}

Service d'Anesthésie Réanimation, CHU de Nantes, 1 place Alexis Ricordeau, 44093 Nantes Cedex 1, France

e-mail: karim.asehnoune@chu-nantes.fr

\section{J. C. Hemphill III}

Departments of Neurology and Neurological Surgery,

University of California, San Francisco, USA

\section{R. S. Agbeko}

Department of Paediatric Intensive Care, Great North Children's Hospital, The Newcastle upon Tyne Hospitals NHS Foundation Trust, Institute of Cellular Medicine, Newcastle University, Newcastle upon Tyne, UK

The management of patients with severe traumatic brain injury (TBI) is at a crossroads. What we know is that there are an estimated 558-790 cases of TBI per 100,000 person-years in industrialized countries annually, costing more than US $\$ 60$ billion each year in the USA alone [1, 2]. We also know that it is bad to have persistently elevated intracranial pressure (ICP) after TBI. What we do not know is how best to treat elevated ICP. Guidelines include ICP monitoring and treatment as part of fundamental tenets [3]. However, recent clinical trials cast doubt on whether it is the ICP management that makes the difference and even whether our standard guidelinesbased approach targeting an ICP level below $20 \mathrm{mmHg}$ is beneficial at all. In the DECRA trial, decompressive craniectomy lowered ICP in TBI patients whose ICP was considered refractory, but did not improve outcome and may have even been harmful [2]. In the BEST-TRIP clinical trial, a strategy of invasive monitoring of ICP with care focused on maintaining ICP below $20 \mathrm{mmHg}$ was not superior to empiric treatment based on solely clinical examination and imaging [4]. It appears that "one size does not fit all" and new approaches in assessing ICP and secondary brain injury are needed [5].

The study by Güiza et al. [6] published in this issue of Intensive Care Medicine describes a new way for assessing the consequences of elevated ICP on outcome after TBI. Using color-coded plots the authors correlated the score on the Glasgow Outcome Scale at 6 months with the number, intensity, and duration of ICP elevations. A transition curve separating the ICP insults associated with good or poor outcome was also described. Interestingly, the cumulative percentage of time above the transition curve was independently associated with mortality. By exploring different ICP thresholds and calculating patient-specific ICP "doses" based on the continuous monitoring of cerebrovascular pressure reactivity, they found that these individualized doses of elevated ICP were stronger predictors of death than doses derived from the standardized thresholds of 20 and $25 \mathrm{mmHg}$. Finally, the authors demonstrated that pediatric TBI patients were more sensitive to these secondary insults than adults. Figure 1 illustrates some of the differences between adults and children.

A major strength of this study is in providing a timebased dose cutpoint regarding the maximal tolerable duration of elevated ICP (above $30 \mathrm{~min}$ approximately) for avoiding poor outcome. Importantly, this study also provides an intuitive, clinician-friendly, visual method of distinguishing potentially harmful states (based on ICP dose) of secondary brain injury. This is not the first study to suggest that a more nuanced and individualized approach to intracranial hypertension may have merit [7]. Vik et al. [8] looked at 135 TBI patients and assessed whether the calculated "ICP dose", estimated as the area under the curve (AUC) of ICP versus time, was related to outcome. They found a significant relationship between 


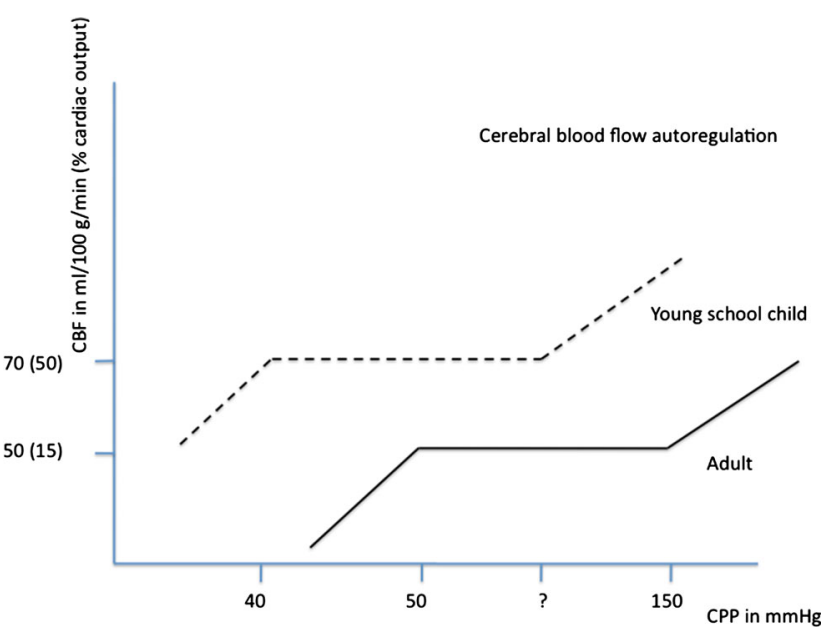

Fig. 1 Relationships between cerebral blood flow (CBF) and cerebral perfusion pressure (CPP). Some of the differences between adults and children. Note the absolute as well as relative higher cerebral blood flow in a young child compared to an adult. Given that cerebral blood flow may be over $50 \%$ of cardiac output in the young child, vs. $15 \%$ in adults, even though the lower inflection point for loss of autoregulation at $40 \mathrm{mmHg}$ in children is lower than in adults (ca. $50 \mathrm{mmHg}$ ), the resultant associated injury may be higher because of a lower capacity to buffer. This is compounded by a higher baseline metabolic rate

the dose of ICP, patient outcome, and the worst Marshall head CT score. The results of Güiza et al. [6] confirm these data by showing that more than a given threshold, the duration of ICP above the treatment goal is probably a critical determinant of patient outcome.

A notable finding of the Guiiza et al. study [6] is the demonstration that when the cerebral perfusion pressure (CPP) was below $50 \mathrm{mmHg}$, any ICP insult was associated with worse outcome. This strongly suggests that the status of pressure autoregulation in an individual patient plays a key role in how dose-insults of ICP are "tolerated." In another study, Lazaridis et al. [9] explored the importance of different ICP thresholds on outcome by calculating patient-specific ICP doses based on the continuous monitoring of cerebrovascular pressure reactivity. These individualized doses of intracranial hypertension were stronger predictors of death than doses derived from the standard threshold of $20 \mathrm{mmHg}$. In the current article by Güiza et al. [6], the association between ICP insults and outcome was examined at three different CPP thresholds $(50,60$, and $70 \mathrm{mmHg})$, with elevated ICP no longer tolerated when CPP was below $50 \mathrm{mmHg}$. Furthermore, there is a suggestion that an ICP threshold of $25 \mathrm{mmHg}$ is not tolerated for more than 10-20 min regardless of the autoregulatory state and thus may be a more appropriate clinical trigger threshold than the more commonly used threshold of $20 \mathrm{mmHg}$. It is relevant to note that the observed association does not mean causality, and as acknowledged by the authors, this direct data visualization will not provide a direct modification of the current ICP thresholds. However, the data presented here [6] seems to give some insight into why randomized clinical trials that use a uniform treatment threshold of $20 \mathrm{mmHg}$ to initiate have failed and frankly may continue to do so [2, 4].

The holy grail of optimal CPP continues to be sought. Recently data were provided to support age-specific CPP goals as greater than 50 or $60 \mathrm{mmHg}$ in adults, greater than $50 \mathrm{mmHg}$ in $6-17$ year olds, and greater than $40 \mathrm{mmHg}$ in $0-5$ year olds [10]. These thresholds may then be combined with individual measures of cerebral blood flow autoregulation, such as measured by the pressure reactivity index (PRx) used in both children [11] and adults [12]. Güiza et al. demonstrate that it is eminently possible to update, integrate, and present data in a way that helps decision-making in our neurocritical care practice.

Finally, this study is noteworthy not just because of the findings described above. We often take data visualization for granted. But a major current problem facing critical care, especially neurocritical care, is data overload. We have lots of data but we are not optimizing the way we collect, analyze, and view it [13]. It may be that Güiza et al. are showing us a glimpse of the future, where we can make better sense of complex relationships between multiple parameters such as ICP and cerebral autoregulation that we would otherwise miss using current approaches to bedside care. The challenges of negative studies in TBI (including those focusing on elevated ICP) have led some to wonder if the emperor is actually naked. Perhaps improved methods of data analysis and visualization such as those described here represent new clothes and will motivate us to become more thoughtful tailors.

\section{Conflicts of interest None.}

\section{References}

1. Lozano R, Naghavi M, Foreman $\mathrm{K}$ et al (2012) Global and regional mortality from 235 causes of death for 20 age groups in 1990 and 2010: a systematic analysis for the Global Burden of Disease Study 2010. Lancet 380:2095-2128. doi:10.1016/ S0140-6736(12)61728-0
2. Cooper DJ, Rosenfeld JV, Murray L et al (2011) Decompressive craniectomy in diffuse traumatic brain injury. N Engl J Med 364:1493-1502. doi:10.1056/NEJMoa1102077
3. Brain Trauma Foundation, American Association of Neurological Surgeons, Congress of Neurological Surgeons (2007) Guidelines for the management of severe traumatic brain injury. J Neurotrauma 24(Suppl 1):S1-S106. doi:10.1089/neu.2007.9999 
4. Chesnut RM, Temkin N, Carney $\mathrm{N}$ et al (2012) A trial of intracranial-pressure monitoring in traumatic brain injury. N Engl J Med 367:2471-2481. doi: 10.1056/NEJMoa1207363

5. Chesnut RM (2013) Intracranial pressure monitoring: headstone or a new head start. The BEST TRIP trial in perspective. Intensive Care Med 39:771-774. doi:10.1007/ s00134-013-2852-9

6. Güiza F, Depreitere B, Piper I, Citerio G, Chambers I, Jones PA, Lo TY, Enblad P, Nillson P, Feyen B, Jorens P, Maas A, Schuhmann MU, Donald R, Moss L, Van den Berghe G, Meyfroidt G (2015) Visualizing the pressure and time burden of intracranial hypertension in adult and paediatric traumatic brain injury. Intensive Care Med. doi: 10.1007/s00134-015-3806-1

7. Stocchetti N, Maas AIR (2014)

Traumatic intracranial hypertension. N Engl J Med 370:2121-2130. doi: 10.1056/NEJMra1208708
8. Vik A, Nag T, Fredriksli OA et al (2008) Relationship of dose of intracranial hypertension to outcome in severe traumatic brain injury.

J Neurosurg 109:678-684. doi: 10.3171/JNS/2008/109/10/0678

9. Lazaridis C, DeSantis SM, Smielewski $P$ et al (2014) Patient-specific thresholds of intracranial pressure in severe traumatic brain injury. J Neurosurg 120:893-900. doi: 10.3171/2014.1.JNS131292

10. Allen BB, Chiu Y-L, Gerber LM et al (2014) Age-specific cerebral perfusion pressure thresholds and survival in children and adolescents with severe traumatic brain injury. Pediatr Crit Care Med 15:62-70. doi:10.1097/PCC. 0b013e3182a556ea

11. Brady KM, Shaffner DH, Lee JK et al (2009) Continuous monitoring of cerebrovascular pressure reactivity after traumatic brain injury in children. Pediatrics 124:e1205-e1212. doi: 10.1542/peds.2009-0550
12. Johnson U, Lewén A, Ronne-Engström E et al (2014) Should the neurointensive care management of traumatic brain injury patients be individualized according to autoregulation status and injury subtype? Neurocrit Care 21:259-265. doi:10.1007/s12028014-9954-2

13. Hemphill JC, Andrews P, De Georgia M (2011) Multimodal monitoring and neurocritical care bioinformatics. Nat Rev Neurol 7:451-460. doi: 10.1038/nrneurol.2011.101 